\title{
Cooperation in patchy environment with cross-diffusion
}

\author{
Aly A. Shaban
}




\title{
COOPERATION IN PATCHY ENVIRONMENT WITH CROSS-DIFFUSION
}

\author{
ALY A. SHABAN
}

[Received: September 16, 2003]

\begin{abstract}
Aвstract. In this paper, we formulate a Lotka-Volterra cooperative system in two patches in which the per capita migration rate of each species is influenced not only by its own but also by the other one's density, i. e., there is cross-diffusion present. Numerical studies show that at a critical value of the bifurcation parameter the system undergoes a Turing bifurcation and the cross-migration response is an important factor that should not be ignored when a pattern emerges.
\end{abstract}

Mathematics Subject Classification: 92A15

Keywords: Cross-diffusion, diffusive instability, pattern formation

\section{InTRODUCTION}

$\mathrm{T}$ He TURING Bifurcation (see [8]) is the basic bifurcation generating a spatial pattern, wherein an equilibrium of a nonlinear system is asymptotically stable in the absence of diffusion but unstable in the presence of diffusion. This lies at the heart of almost all mathematical models for patterning in ecology, embryology, and elsewhere in biology and chemistry (see $[1,2]$ ). Since the relation between organisms and space seems to be essential to stability of an ecological system, the effect of diffusion on the possibility of species coexistence in an ecological community has been an important subject in population biology (see [5-8]). We consider a two-species cooperative Lotka-Volterra system living in a habitat of two identical patches linked by migration and we show that at a critical value of the bifurcation parameter the system undergoes a Turing bifurcation, i. e., the stable constant steady state loses its stability and spatially non-constant stationary solutions, a pattern emerges.

This paper is organized as follows. In Section 2, the model is built, in Section 3 its linearization is treated and the conditions for the Turing bifurcation are established (these are the main results of the paper), in Section 4 we consider an example to illustrate what can be expected, and in Section 5 we summarize the main conclusions of the study.

Research partially supported by the Hungarian National Foundation for Scientific Research. 


\section{THE MODEL}

We consider a two-species cooperative Lotka-Volterra system living in a habitat of two identical patches linked by migration.

Let $u_{i}(t, j)$ be the density of species $i$ in patch $j$ at time $t, i=1,2 ; j=1,2$; $t \in \mathbb{R}$. The cooperation between two species is described by the system of differential equations

$$
\begin{aligned}
\dot{u}_{1}(t, 1) & =u_{1}(t, 1)\left(r_{1}-a_{11} u_{1}(t, 1)-a_{12} u_{2}(t, 1)\right) \\
& +d_{1}\left(\varrho_{1}\left(u_{2}(t, 2)\right) u_{1}(t, 2)-\varrho_{1}\left(u_{2}(t, 1)\right) u_{1}(t, 1)\right), \\
\dot{u}_{2}(t, 1) & =u_{2}(t, 1)\left(r_{2}-a_{21} u_{1}(t, 1)-a_{22} u_{2}(t, 1)\right) \\
& +d_{2}\left(\varrho_{2}\left(u_{1}(t, 2)\right) u_{2}(t, 2)-\varrho_{2}\left(u_{1}(t, 1)\right) u_{2}(t, 1)\right), \\
\dot{u}_{1}(t, 2) & =u_{1}(t, 2)\left(r_{1}-a_{11} u_{1}(t, 2)-a_{12} u_{2}(t, 2)\right) \\
& +d_{1}\left(\varrho_{1}\left(u_{2}(t, 1)\right) u_{1}(t, 1)-\varrho_{1}\left(u_{2}(t, 2)\right) u_{1}(t, 2)\right), \\
\dot{u}_{2}(t, 2) & =u_{2}(t, 2)\left(r_{2}-a_{21} u_{1}(t, 2)-a_{22} u_{2}(t, 2)\right) \\
& +d_{2}\left(\varrho_{2}\left(u_{1}(t, 1)\right) u_{2}(t, 1)-\varrho_{2}\left(u_{1}(t, 2)\right) u_{2}(t, 2)\right),
\end{aligned}
$$

where

$$
a_{11}, a_{22}>0, \quad a_{12}, a_{21}<0, \quad d_{i} \geq 0, \quad \varrho_{i}>0 \quad \text { for } i, k=1,2 .
$$

Here, $a_{11}$ and $a_{22}$ represent the strength of the intraspecific competition (the competition within the species, $\frac{r_{1}}{a_{11}}, \frac{r_{2}}{a_{22}}$ are the carrying capacities for the respective species), $\left|a_{12}\right|$ and $\left|a_{21}\right|$ represent the strength of interspecific cooperation, $d_{i}>0(i=1,2)$ are the diffusion coefficients, and $\varrho_{1} \in C^{1}$ is a positive decreasing function of $u_{2}$, with analogous conditions on $\varrho_{2}$. The idea is that these migration functions describe the inclination of individuals of one species to stay at a certain patch due to the attraction by the other species in the patch (see $[1,3,4])$.

The case to be considered is where each species survives if left alone and follows the logistic dynamics, that is, the intrinsic growth rates of the respective species are positive, $r_{1}, r_{2}>0$, which is called facultative cooperation.

We consider the kinetic system without migration, i. e., the case where $d_{1}$ and $d_{2}$ are equal to zero:

$$
\begin{aligned}
& \dot{u}_{1}(t, 1)=u_{1}(t, 1)\left(r_{1}-a_{11} u_{1}(t, 1)-a_{12} u_{2}(t, 1)\right), \\
& \dot{u}_{2}(t, 1)=u_{2}(t, 1)\left(r_{2}-a_{21} u_{1}(t, 1)-a_{22} u_{2}(t, 1)\right), \\
& \dot{u}_{1}(t, 2)=u_{1}(t, 2)\left(r_{1}-a_{11} u_{1}(t, 2)-a_{12} u_{2}(t, 2)\right), \\
& \dot{u}_{2}(t, 2)=u_{2}(t, 2)\left(r_{2}-a_{21} u_{1}(t, 2)-a_{22} u_{2}(t, 2)\right) .
\end{aligned}
$$

We assume that

$$
\operatorname{det} A=a_{11} a_{22}-a_{21} a_{12}>0 .
$$

Then system (2) has a positive equilibrium

$$
\left(u_{1}(t, 1), u_{1}(t, 2), u_{2}(t, 1), u_{2}(t, 2)\right) \equiv\left(\bar{u}_{1}, \bar{u}_{2}, \bar{u}_{1}, \bar{u}_{2}\right),
$$


where

$$
\bar{u}_{1}=\frac{r_{1} a_{22}-r_{2} a_{12}}{\operatorname{det} A}, \quad \bar{u}_{2}=\frac{r_{2} a_{11}-r_{1} a_{21}}{\operatorname{det} A} .
$$

The Jacobian matrix of the system without diffusion linearized at $\left(\bar{u}_{1}, \bar{u}_{2}, \bar{u}_{1}, \bar{u}_{2}\right)$ is

$$
J_{k}=\left(\begin{array}{cccc}
-a_{11} \bar{u}_{1} & -a_{12} \bar{u}_{1} & 0 & 0 \\
-a_{21} \bar{u}_{2} & -a_{22} \bar{u}_{2} & 0 & 0 \\
0 & 0 & -a_{11} \bar{u}_{1} & -a_{12} \bar{u}_{1} \\
0 & 0 & -a_{21} \bar{u}_{2} & -a_{22} \bar{u}_{2}
\end{array}\right) .
$$

The corresponding characteristic polynomial has the form

$$
D_{4}(\lambda)=\left(D_{2}(\lambda)\right)^{2}, \quad D_{2}(\lambda)=\lambda^{2}+\lambda\left(a_{11} \bar{u}_{1}+a_{22} \bar{u}_{2}\right)+\bar{u}_{1} \bar{u}_{2} \operatorname{det} A .
$$

Since $a_{11} \bar{u}_{1}+a_{22} \bar{u}_{2}>0$ and $\operatorname{det} A>0$, we see that the coexistence equilibrium point $\left(\bar{u}_{1}, \bar{u}_{2}, \bar{u}_{1}, \bar{u}_{2}\right)$ is linearly asymptotically stable.

\section{The LiNEARIZED PROBLEM}

Returning to system (2.1), we see that $\left(\bar{u}_{1}, \bar{u}_{2}, \bar{u}_{1}, \bar{u}_{2}\right)$ is also a spatially homogeneous equilibrium of the system with diffusion. The Jacobian matrix of the system with diffusion at $\left(\bar{u}_{1}, \bar{u}_{2}, \bar{u}_{1}, \bar{u}_{2}\right)$ is written as

$$
J_{D}=\left(\begin{array}{cccc}
-a_{11} \bar{u}_{1}-d_{1} \varrho_{1} & -a_{12} \bar{u}_{1}-d_{1} \varrho_{1}^{\prime} \bar{u}_{1} & d_{1} \varrho_{1} & d_{1} \varrho_{1}^{\prime} \bar{u}_{1} \\
-a_{21} \bar{u}_{2}-d_{2} \varrho_{2}^{\prime} \bar{u}_{2} & -a_{22} \bar{u}_{2}-d_{2} \varrho_{2} & d_{2} \varrho_{2}^{\prime} \bar{u}_{2} & d_{2} \varrho_{2} \\
d_{1} \varrho_{1} & d_{1} \varrho_{1}^{\prime} \bar{u}_{1} & -a_{11} \bar{u}_{1}-d_{1} \varrho_{1} & -a_{12} \bar{u}_{1}-d_{1} \varrho_{1}^{\prime} \bar{u}_{1} \\
d_{2} \varrho_{2}^{\prime} \bar{u}_{2} & d_{2} \varrho_{2} & -a_{21} \bar{u}_{2}-d_{2} \varrho_{2}^{\prime} \bar{u}_{2} & -a_{22} \bar{u}_{2}-d_{2} \varrho_{2}
\end{array}\right)
$$

where $\varrho_{1}$ and $\varrho_{1}^{\prime}$ are to be taken at $\bar{u}_{2}$ and $\varrho_{2}, \varrho_{2}^{\prime}$ at $\bar{u}_{1}$. We have

$$
\operatorname{det}\left(J_{D}-\lambda I\right)=\left|\begin{array}{cccc}
-a_{11} \bar{u}_{1}-d_{1} \varrho_{1}-\lambda-a_{12} \bar{u}_{1}-d_{1} \varrho_{1}^{\prime} \bar{u}_{1} & d_{1} \varrho_{1} & d_{1} \varrho_{1}^{\prime} \bar{u}_{1} \\
-a_{21} \bar{u}_{2}-d_{2} \varrho_{2}^{\prime} \bar{u}_{2} & -a_{22} \bar{u}_{2}-d_{2} \varrho_{2}-\lambda & d_{2} \varrho_{2}^{\prime} \bar{u}_{2} & d_{2} \varrho_{2} \\
d_{1} \varrho_{1} & d_{1} \varrho_{1}^{\prime} \bar{u}_{1} & -a_{11} \bar{u}_{1}-d_{1} \varrho_{1}-\lambda & -a_{12} \bar{u}_{1}-d_{1} \varrho_{1}^{\prime} \bar{u}_{1} \\
d_{2} \varrho_{2}^{\prime} \bar{u}_{2} & d_{2} \varrho_{2} & -a_{21} \bar{u}_{2}-d_{2} \varrho_{2}^{\prime} \bar{u}_{2} & -a_{22} \bar{u}_{2}-d_{2} \varrho_{2}-\lambda
\end{array}\right| .
$$


Using the properties of determinant, we get

$$
\begin{aligned}
\operatorname{det}\left(J_{D}-\lambda I\right)= & \left|\begin{array}{cccc}
-a_{11} \bar{u}_{1}-\lambda & -a_{12} \bar{u}_{1} & d_{1} \varrho_{1} & d_{1} \varrho_{1}^{\prime} \bar{u}_{1} \\
-a_{21} \bar{u}_{2} & -a_{22} \bar{u}_{2}-\lambda & d_{2} \varrho_{2}^{\prime} \bar{u}_{2} & d_{2} \varrho_{2} \\
0 & 0 & -a_{11} \bar{u}_{1}-2 d_{1} \varrho_{1}-\lambda & -a_{12} \bar{u}_{1}-2 d_{1} \varrho_{1}^{\prime} \bar{u}_{1} \\
0 & 0 & -a_{21} \bar{u}_{2}-2 d_{2} \varrho_{2}^{\prime} \bar{u}_{2} & -a_{22} \bar{u}_{2}-2 d_{2} \varrho_{2}-\lambda
\end{array}\right| \\
= & D_{2}(\lambda)\left(\lambda^{2}+\lambda\left(a_{11} \bar{u}_{1}+a_{22} \bar{u}_{2}+2\left(d_{1} \varrho_{1}+d_{2} \varrho_{2}\right)\right)+\bar{u}_{1} \bar{u}_{2} \operatorname{det} A\right. \\
& +2 \bar{u}_{1} d_{2}\left(a_{11} \varrho_{2}-a_{12} \varrho_{2}^{\prime} \bar{u}_{2}\right)+2 \bar{u}_{2} d_{1}\left(a_{22} \varrho_{1}-a_{21} \varrho_{1}^{\prime} \bar{u}_{1}\right) \\
& \left.+4 d_{1} d_{2}\left(\varrho_{1} \varrho_{2}-\bar{u}_{1} \bar{u}_{2} \varrho_{1}^{\prime} \varrho_{2}^{\prime}\right)\right) .
\end{aligned}
$$

We know that $D_{2}(\lambda)$ has two roots with negative real parts. The other polynomial will have a negative and a positive root if the constant term is negative. Clearly,

$$
\left(\varrho_{1} \varrho_{2}-\bar{u}_{1} \bar{u}_{2} \varrho_{1}^{\prime} \varrho_{2}^{\prime}\right)=\varrho_{1} \varrho_{2}\left(1-\bar{u}_{1} \bar{u}_{2} \frac{\varrho_{1}^{\prime} \varrho_{2}^{\prime}}{\varrho_{1} \varrho_{2}}\right)<0
$$

if $\frac{\varrho_{1}^{\prime} \varrho_{2}^{\prime}}{\varrho_{1} \varrho_{2}}$ is large enough. If we have achieved this, we may increase $d_{1}$ and/or $d_{2}$ and the constant term becomes negative (see [3]). These calculations lead us to the following theorem.

Theorem. The equilibrium $\left(\bar{u}_{1}, \bar{u}_{2}, \bar{u}_{1}, \bar{u}_{2}\right)$ of system (2.1) is asymptotically stable if $\frac{\varrho_{1}^{\prime} \varrho_{2}^{\prime}}{\varrho_{1} \varrho_{2}}, d_{1}$ and $d_{2}$ are sufficiently small. If $\frac{\varrho_{\varrho_{1}^{\prime}}^{\prime} \varrho_{2}^{\prime}}{\varrho_{1} \varrho_{2}}$ and either $d_{1}$ or $d_{2}$ are sufficiently large, then $\left(\bar{u}_{1}, \bar{u}_{2}, \bar{u}_{1}, \bar{u}_{2}\right)$ loses its stability by a Turing bifurcation.

Remark. The situation is different if the cooperation is obligatory, $r_{1}, r_{2}<0$; the condition of having a point of intersection in the positive quadrant is

$$
\operatorname{det} A=a_{11} a_{22}-a_{21} a_{12}<0 .
$$

The characteristic polynomial of the linearized system (2.6) without diffusion at $\left(\bar{u}_{1}, \bar{u}_{2}, \bar{u}_{1}, \bar{u}_{2}\right)$ has the form

$$
D_{4}(\lambda)=\left(D_{2}(\lambda)\right)^{2}, \quad D_{2}(\lambda)=\lambda^{2}+\lambda\left(a_{11} \bar{u}_{1}+a_{22} \bar{u}_{2}\right)+\bar{u}_{1} \bar{u}_{2} \operatorname{det} A .
$$

Since $a_{11} \bar{u}_{1}+a_{22} \bar{u}_{2}>0$ and $\operatorname{det} A<0$, we see that the coexistence equilibrium point $\left(\bar{u}_{1}, \bar{u}_{2}, \bar{u}_{1}, \bar{u}_{2}\right)$ is a saddle point and diffusion never stabilizes an equilibrium which is unstable for the kinetic system.

\section{NuMERICAL INVESTIGATION}

In this section, we apply our analytical approach of Section 3 to a specific migration function and we are looking for conditions which imply the Turing instability (diffusion driven instability). Namely, we choose

$$
\varrho_{1}\left(u_{2}\right)=m_{1} \exp \left(-u_{2} / m_{1}\right), \quad \varrho_{2}\left(u_{1}\right)=m_{2} \exp \left(-u_{1} / m_{2}\right), \quad m_{1}, m_{2}>0 .
$$


If $r_{1}=2, r_{2}=1, a_{11}=5, a_{22}=4, a_{12}=-4, a_{21}=-3, m_{1}=1, m_{2}=1$, and $d_{1}=1$, then

$$
\operatorname{det} A=a_{11} a_{22}-a_{21} a_{12}=8
$$

and

$$
\bar{u}_{1}=\frac{r_{1} a_{22}-r_{2} a_{12}}{\operatorname{det} A}=\frac{3}{2}, \quad \bar{u}_{2}=\frac{r_{2} a_{11}-r_{1} a_{21}}{\operatorname{det} A}=\frac{11}{8} .
$$

At $d_{2}=d_{2 \text { crit }}$,

$$
d_{2 \text { crit }}=\frac{-\frac{33}{2}+\frac{11}{8 \exp \left(\frac{11}{8}\right)}}{-\frac{17}{4 \exp \left(\frac{23}{8}\right)}-\frac{3}{2 \exp \left(\frac{3}{2}\right)}} \cong 28.11725408,
$$

we have four eigenvalues $\lambda_{i}(i=1,2,3,4)$ such that $\lambda_{i}<0(i=1,2,3)$ and $\lambda_{4}=0$.

\begin{tabular}{|c|c|c|c|c|}
\hline$d_{2}$ & $u_{1}(t, 1)$ & $u_{2}(t, 1)$ & $u_{1}(t, 2)$ & $u_{2}(t, 2)$ \\
\hline \hline 28 & 1.500000000 & 1.375000000 & 1.500000000 & 1.375000000 \\
\hline \multirow{3}{*}{28.2} & 1.524440807 & 1.403943678 & 1.474516967 & 1.344807960 \\
& 1.500000000 & 1.375000000 & 1.500000000 & 1.375000000 \\
& 1.474516967 & 1.344807960 & 1.524440807 & 1.403943678 \\
\hline \multirow{3}{*}{29} & 1.574289053 & 1.462917090 & 1.415068046 & 1.274335600 \\
& 1.500000000 & 1.375000000 & 1.500000000 & 1.375000000 \\
& 1.415068046 & 1.274335600 & 1.574289053 & 1.462917090 \\
\hline \multirow{3}{*}{30} & 1.602257674 & 1.495956494 & 1.376189993 & 1.228230906 \\
& 1.500000000 & 1.375000000 & 1.500000000 & 1.375000000 \\
& 1.376189993 & 1.228230906 & 1.602257674 & 1.495956494 \\
\hline \multirow{3}{*}{50} & 1.683294096 & 1.591314489 & 1.225824281 & 1.049861530 \\
& 1.500000000 & 1.375000000 & 1.500000000 & 1.375000000 \\
& 1.225824281 & 1.049861530 & 1.683294096 & 1.591314489 \\
\hline \multirow{3}{*}{50} & 1.704327936 & 1.615899482 & 1.169559919 & .9830950704 \\
& 1.500000000 & 1.375000000 & 1.500000000 & 1.375000000 \\
& 1.169559919 & .9830950704 & 1.704327936 & 1.615899482 \\
\hline \multirow{3}{*}{60} & 1.714081033 & 1.627253651 & 1.138343964 & .9460399293 \\
& 1.500000000 & 1.375000000 & 1.500000000 & 1.375000000 \\
& 1.138343964 & .9460399293 & 1.714081033 & 1.627253651 \\
\hline \multirow{3}{*}{80} & 1.723234442 & 1.637870036 & 1.104276166 & .9055833674 \\
& 1.500000000 & 1.500000000 & 1.500000000 & 1.500000000 \\
& 1.104276166 & .9055833674 & 1.723234442 & 1.637870036 \\
\hline \multirow{3}{*}{100} & 1.727536907 & 1.642841684 & 1.085923814 & .8837801086 \\
& 1.500000000 & 1.500000000 & 1.500000000 & 1.500000000 \\
& 1.085923814 & .8837801086 & 1.727536907 & 1.642841684 \\
\hline
\end{tabular}

TABLE 1. The equilibria before and after bifurcation (Section 4). 
If $d_{2}<d_{2 \text { crit }}$, then $\lambda_{i}<0(i=1,2,3,4)$, and $\left(\bar{u}_{1}, \bar{u}_{2}, \bar{u}_{1}, \bar{u}_{2}\right)$ is asymptotically stable. If $d_{2}>d_{2 \text { crit }}$, then $\lambda_{i}<0(i=1,2,3)<0$ and $\lambda_{4}>0$; hence, $\left(\bar{u}_{1}, \bar{u}_{2}, \bar{u}_{1}, \bar{u}_{2}\right)$ is unstable in this case.

Thus, as $d_{2}$ is increased through $d_{2}=d_{2 \text { crit }}$, then the spatially homogeneous equilibrium loses its stability. Numerical calculations show that two new spatially nonconstant equilibria emerge, and these equilibria are asymptotically stable, so that this is a pitchfork bifurcation. Table 1 contains some numerical data, whereas Figure 2 shows the graphs* of the coordinate $u_{1}(t, 1)$ of solutions corresponding to the respective initial conditions

$(1.80,1.60,1.50,1.25),(1.20,1.10,1.59,1.47),(1.58,1.45,1.36,1.22)$,

$(1.00,1.10,1.585,1.47),(1.65,1.100,1.320,1.500)$

in the case where $d_{2}=30$. The three solutions on Figure 2 tend to 1.6022576 and two solutions tend to 1.376189 . Figure 1 shows the corresponding solutions in a "pre-bifurcation case" (for $d_{2}=28$ ).

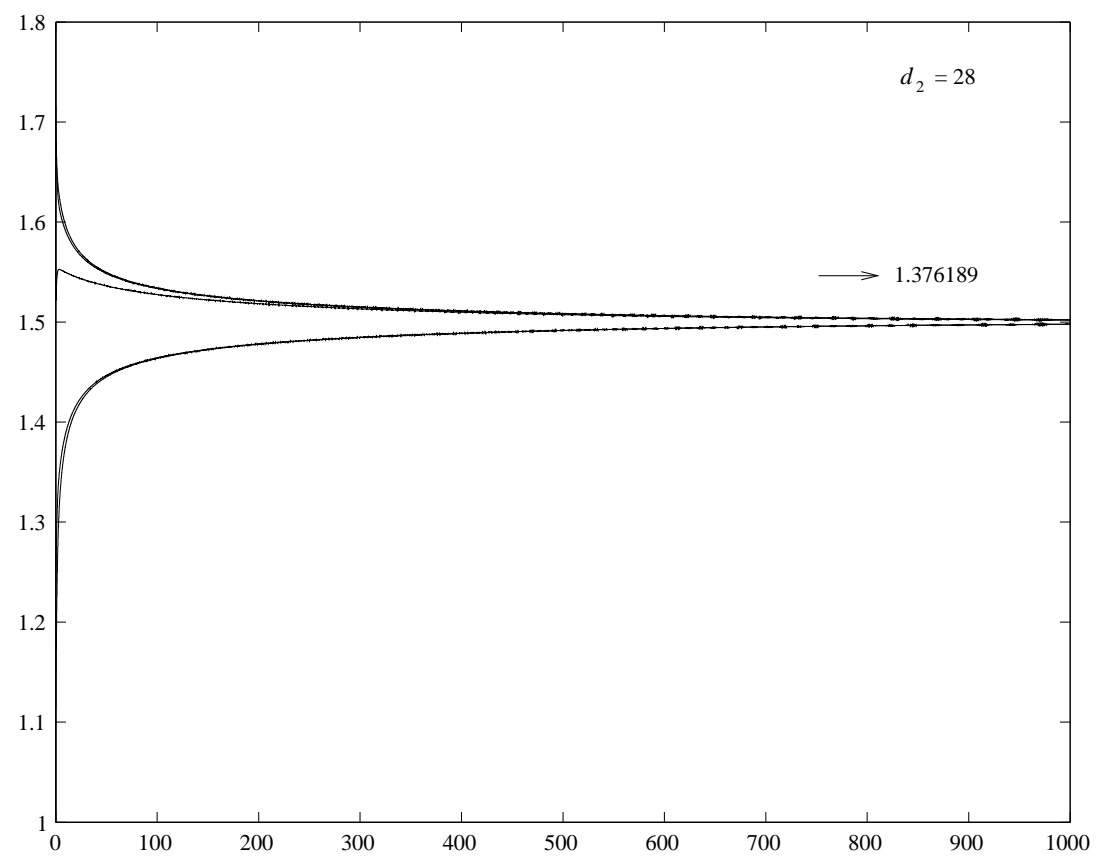

FIGURE 1. Graphs of the coordinate $u_{1}(t, 1)$ of the solutions in a "prebifurcation case" (Section 4, $d_{2}=28$ )

${ }^{*}$ The graphs were produced by using PHASER. 


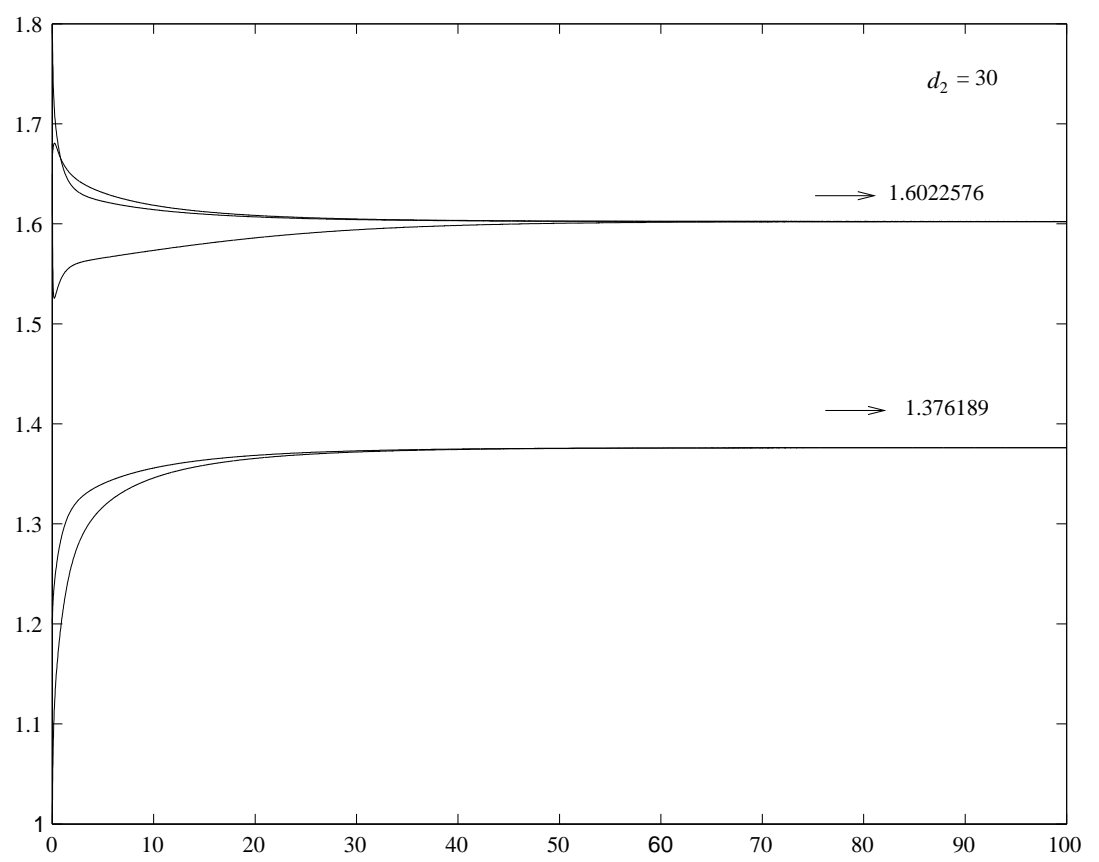

Figure 2. Graphs of the coordinate $u_{1}(t, 1)$ of the five solutions (Section $4, d_{2}=30$; see Table 1) corresponding to the respective initial conditions (4.2).

It should be noted that, after the bifurcation, the sum of the stable equilibrium values of species 1 at the two patches (and, similarly, that of species 2) is equal to the double of its spatially homogeneous equilibrium value $\bar{u}_{1}$ (resp., $\bar{u}_{2}$ ).

\section{Conclusions}

In the present paper, our interest is to study a Lotka-Volterra cooperative system in two patches in which the per capita migration rate of each species is influenced not only by its own but also by the other one's density, i. e., there is cross-diffusion present. We show that, at a critical value of the bifurcation parameter, the system undergoes a Turing bifurcation, and the cross-migration response is an important factor that should not be ignored when a pattern emerges. As $d_{2}$ is increased through $d_{2}=d_{2 \text { crit }}$, the spatially homogeneous equilibrium loses its stability, and two new stable equilibria emerge. Diffusion never stabilizes an equilibrium which is unstable for the kinetic system. 


\section{REFERENCES}

[1] Farkas, M.: Two ways of modeling cross diffusion, Nonlinear Analysis. Theory, Methods \& Appl., 30 (1997), 1225-1233.

[2] Farkas, M.: Dynamical Models in Biology, Academic Press, San Diego, 2001.

[3] Huang, Y. and Diekmann, O.: Interspecific influence on mobility and Turing instability, Bull. Math. Biol., 65 (2003), 143-156.

[4] Jansen, V. A. A. AND Alun Lloyd, L.: Local stability analysis of spatially homogeneous solutions of multi-patch systems, J. Math. Biol., 41 (2000), 232-252.

[5] Murray, J. D.: Mathematical Biology, Springer-Verlag, Berlin 1989.

[6] Shaban, A. and Farkas, M.: Competition in patchy environment with cross-diffusion, submitted.

[7] Tакеuchi, Y.: Global Dynamical Properties of Lotka-Volterra Systems, World Scientific, River Edge, NJ, 1996.

[8] Turing, A. M.: The chemical basis of morphogenesis, Philos. Trans. Roy. Soc. London, B237 (1953) 37-72; reprinted in Bull. Math. Biol., 52 (1990), 153-197.

\section{Author's Address}

Aly A. Shaban:

Institute of Mathematics, Budapest University of Technology, Budapest, Hungary, H-1521

E-mail address: shaban@math. bme.hu 